\title{
TRATAMENTO DE FASCEÍTE NECROTIZANTE PERINEAL (SÍNDROME DE FOURNIER) COM HYCOS ${ }^{\circledR}$
}

\section{NECROTIZING FASCIITIS: TREATMENT WITH CREAM HYCOS®}

\section{Karina Vanzan Maio Rodrigues ${ }^{1}$ * Izabel de Almada ${ }^{2}$ * Ana Paula Rodrigues Silva ${ }^{3}$ * Jéssika Costa da Silva ${ }^{4}$ * Rafael Martins Barbosa Campos ${ }^{5}$ * Maria da Conceição Baldini Benevides Blanck $^{6}$}

\section{INTRODUÇÃO}

A síndrome de Fournier $^{1}$ foi descrita em 1883 por Jean Alfred Fournier, como uma infecção de rápida progressão do períneo e da parede abdominal que se estendia para bolsa escrotal e pênis, através da fáscia de Dartos no homem. Fournier caracterizou a síndrome por 3 sinais: início súbito em jovens, saudáveis, com rápida progressão e ausência de agente etiológico específico.

Pode ser idiopática ou associada à comorbidades $^{2}$, como: estados debilitantes (desnutrição, sepse) ou imunossupressores (diabetes mellitus, alcoolismo crônico, doença maligna subjacente, AIDS, uso de quimioterápicos, leucemias), cirurgias colorretais e urogenitais (pós-operatório com uso de instrumentação urológica), uso de drogas endovenosas e trauma (local, mecânico) $)^{3}$.
As bactérias que atingem o subcutâneo causam endarterite obliterativa nos vasos da região escrotal e perineal, gerando necrose. A combinação de edema, inflamação e infecção diminuem o suprimento sanguíneo local e a hipóxia predispondo ao crescimento de microorganismos anaeróbios. $\mathrm{O}$ metabolismo anaeróbico destas bactérias resulta na produção de gás, que se acumula no subcutâneo, causando a crepitação à palpação, observados ao exame físico ${ }^{4}$.

Em pacientes idosos, a fasceíte pode ser silenciosa, enquanto que, em adultos jovens, o quadro séptico é mais comum. $\mathrm{O}$ tratamento inclui desbridamento, extenso, antibioticoterapia de largo espectro $^{5}$ e oxigenioterapia hiperbárica ${ }^{6}$, para tratar a infecção; e preparar o leito para a reconstrução da região perineal e peniana.

\footnotetext{
${ }^{1}$ Médica formada pela UGF, Residência em Cirurgia Geral na Santa Casa de Misericórdia do RJ, Especialista em cirurgia Plástica pela SBCP e Mec, Membro titular da SBCP, Membro da ISAPS, Médica cirurgia plástica do HMSF, Staff do Ctq do HTO da Baixada. Presidente da comissão de curativos do HMSF. Orcid: https://orcid.org/0000-0002-2669-3437

${ }^{2}$ Enfermeira da Comissão de Curativos do Hospital Municipal Salgado Filho. Presidente da Comissão de Curativos do HMSF. Orcid: https://orcid.org/0000-0003-2838-0490.

${ }^{3}$ Residente Curso de Pós-Graduação em nível de Especialização sob a forma de Treinamento em Serviço para Enfermeiros Unidades de Saúde da Rede Municipal de Saúde. Orcid: https://orcid.org/0000-0003-0859-7385

${ }^{4}$ Residente Curso de Pós-Graduação em nível de Especialização sob a forma de Treinamento em Serviço para Enfermeiros Unidades de Saúde da Rede Municipal de Saúde. Orcid: https://orcid.org/0000-0002-4271-4461

${ }^{5}$ Residente Curso de Pós-Graduação em nível de Especialização sob a forma de Treinamento em Serviço para Enfermeiros Unidades de Saúde da Rede Municipal de Saúde. Orcid: https://orcid.org/0000-0002-9689-1631

${ }^{6}$ Enfermeira. Especialista em úlcera por pressão e feridas, doutoranda em Saúde Pública pela UCES- Argentina, Presidente da Sociedade Brasileira de Feridas e Estética (SOBENFeE) e da Sociedade Ibero-Latino-Americana em Úlceras e Feridas (SILAUHE), e membro do grupo técnico de feridas do SUS/MS. Orcid: https://orcid.org/0000-0001-8879-0403
} 
A etiologia da doença geralmente é polimicrobiana $^{7}$, com flora mista na maioria dos casos, na qual podemos encontrar bactérias Gram negativas (Escherichia coli, Proteus mirabilis, Klebsiella $s p$, Pseudomonas, Bacteroides, Acinetobacter $s p$ ), bactérias Gram positivas (Estafilococos, Estreptococos, Enterococos, Clostridium) e fungos.

\section{RELATO DE CASO}

J.Q.V., sexo masculino, 75 anos, Hipertenso, Diabético, etilista crônico, cardiopata grave, com câncer avançado de próstata que interrompeu início de tratamento devido a pandemia de Coronavírus 19.
Deu entrada na unidade na emergência com relato de dor e edema na bolsa escrotal há algumas semanas.

\section{Dia 24/08/2020}

Internou na Enfermaria da Cirurgia Geral após desbridamento cirúrgico de lesão perineal, iniciou esquema de ATB ciprofloxacino 400mg 12/12h + clindamicina 600mg 6/6h.

Após quatro dias, no dia 28 de agosto de 2020, realizou uma nova abordagem cirúrgica, devido a grande quantidade de necrose e esfacelos, sendo desbridada toda bolsa escrotal, ficando com testículos expostos.

\section{Dia 29/08/2020}

Figura 1 - Lesão Perineal 1

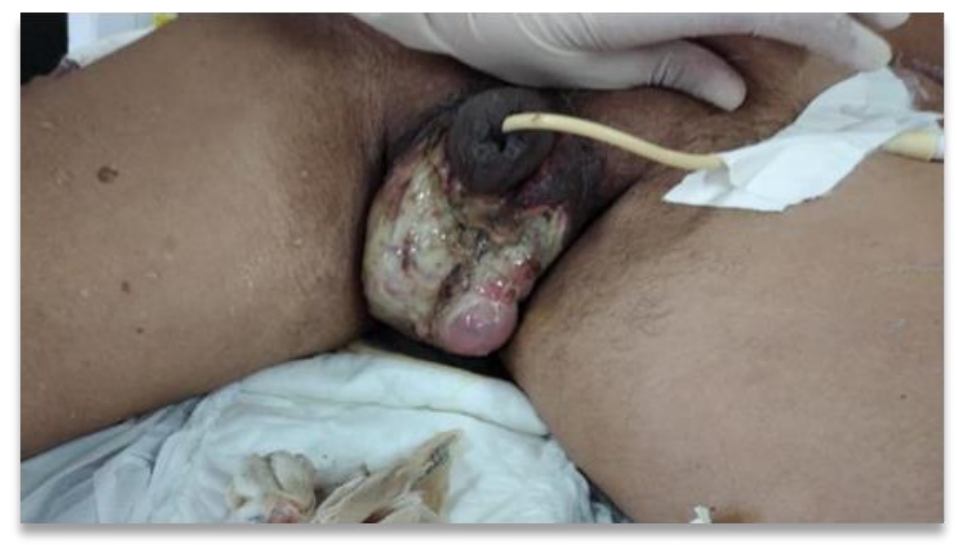

Fonte: Arquivo pessoal
Figura 2 - HYCOS

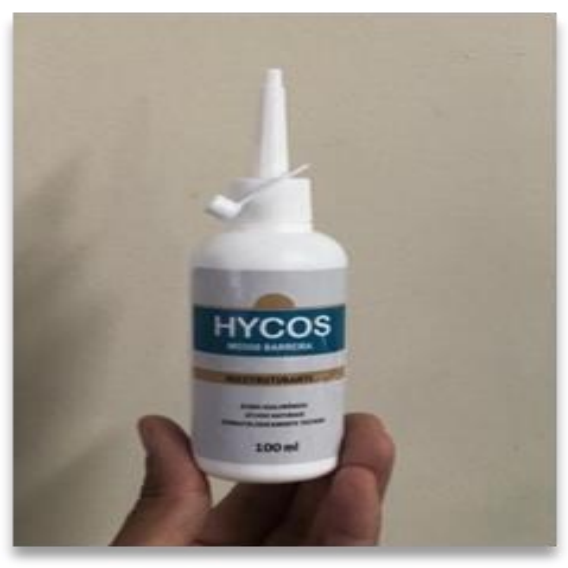

Fonte: Arquivo pessoal
Iniciou o uso do HYCOS na lesão, após 2 desbridamentos, utilizando o creme uma vez ao dia. É um creme reestruturante com Ácido Hialurônico, que tem papel fundamental no tecido em regeneração e angiogênese. Contém ativos naturais com propriedades cicatrizantes, anti-inflamatórias e antissépticas, bactericidas e germicidas, sendo um produto natural de toxicidade zero ${ }^{8}$. 
Dia 02/09/2020

$4^{\circ}$ dia de HYCOS

Figura 2 - Lesão Perineal 2

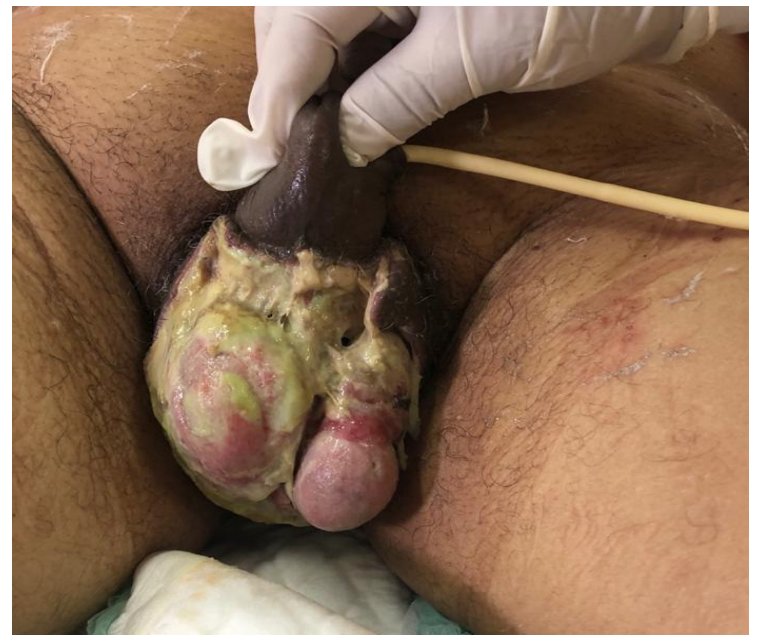

Fonte: Arquivo pessoal

\section{Dia 08/09/2020}

\section{$10^{\circ}$ dia de HYCOS}

Figura 3 - Lesão Perineal 3

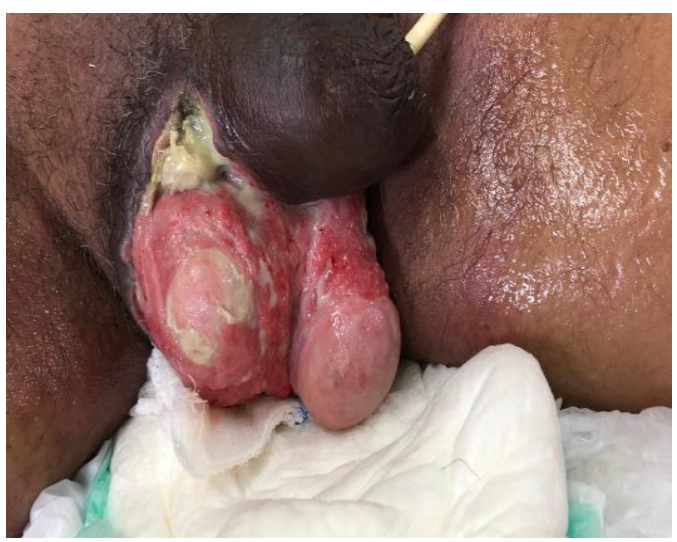

Fonte: Arquivo pessoal

Dia 10/09/2020

\section{$12^{\circ}$ dia de HYCOS}

No dia 12 de setembro de 2020, o paciente foi reavaliado pela cirurgia Plástica que identificou a melhora importante da lesão e orientou alta hospitalar para tratamento ambulatorial. 
Figura 4 - Lesão Perineal 4

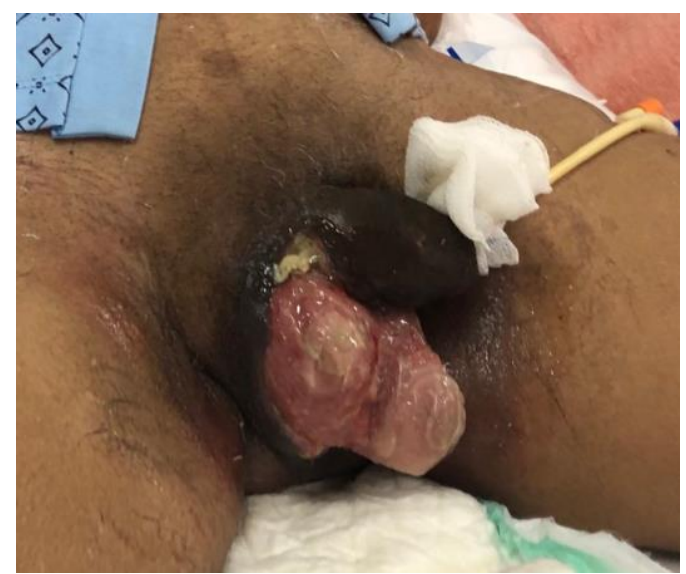

Fonte: Arquivo pessoal

Dia 01/02/2021

Figura 5 - Lesão Perineal 5

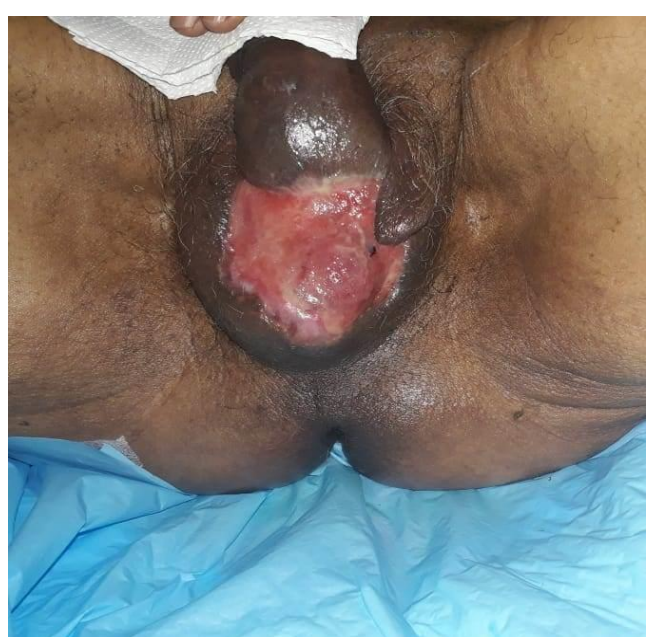

Fonte: Arquivo pessoal

Dia 10/2/2021

Figura 7 - Lesão Perineal 7

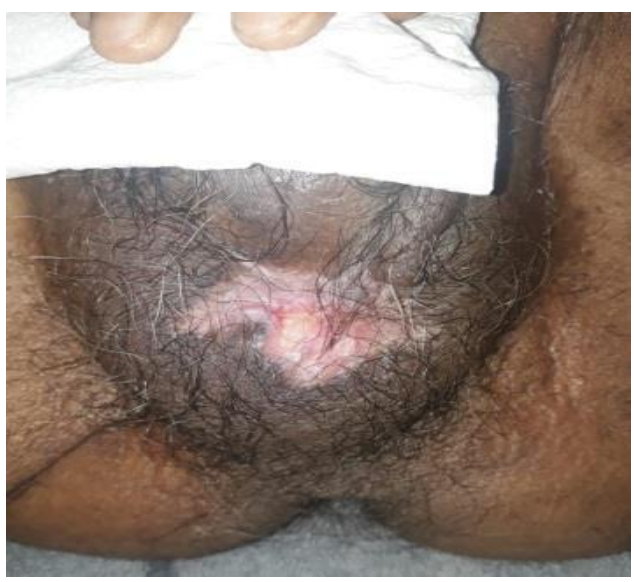

Fonte: Arquivo pessoal
Dia 08/02/2021

Figura 6 - Lesão Perineal 6

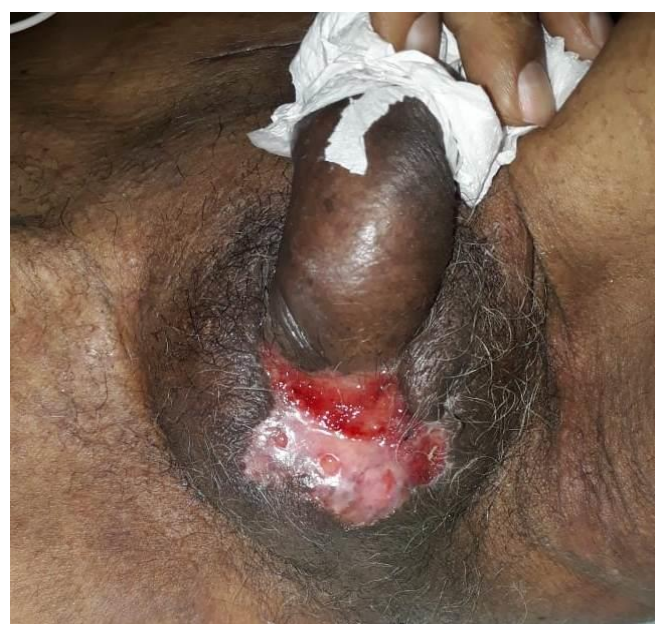

Fonte: Arquivo pessoal

\section{Dia $12 / 02 / 2021$}

Figura 8 - Lesão Perineal 8

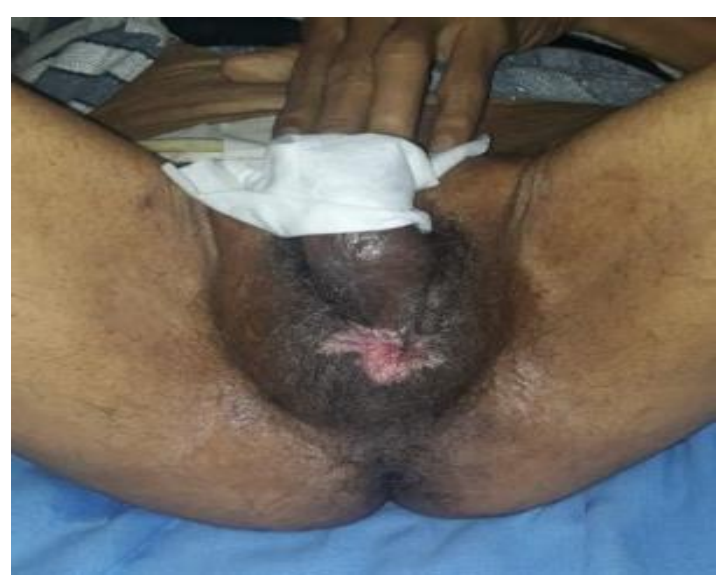

Fonte: Arquivo pessoal 
Ferida completamente cicatrizada por contração das bordas e epitelização e paciente em tratamento quimioterápico do $\mathrm{CA}$ de próstata.

\section{DISCUSSÃO}

A síndrome de Fournier é uma doença com elevada taxa de morbidade e mortalidade e sua evolução esta diretamente relacionada à abordagem precoce e agressiva da lesão . Existem diversos fatores que são predisponentes e agravantes. No caso apresentado haviam diversos fatores agravantes, iniciando pela idade avançada, diabetes, hipertensão, cardiopatia grave e doença neoplásica, porém mesmo assim o tratamento avançou de forma satisfatória e conservadora. Não foi realizada reconstrução cirúrgica da ferida na bolsa escrotal e no períneo, uma vez que o paciente não obteve liberação através do risco cirúrgico, pelo cardiologista, devido as múltiplas comorbidades $^{9,10}$.

Sendo assim foi tratado conservadoramente com a pomada HYCOS que promoveu total granulação e epitelização da ferida.

\section{CONCLUSÃO}

A síndrome de Fournier é uma doença grave e necessita de abordagem rápida. Algumas vezes devido as comorbidades, fica difícil submeter o paciente a múltiplos desbridamentos, uma internação prolongada e ao mesmo tempo conseguir realizar uma reconstrução cirúrgica definitiva da área.

A pomada HYCOS surge como facilitador do processo, promovendo desbridamento autolítico da área pelo extrato de Carica papaya e ao mesmo tempo promovendo a cicatrização da ferida, através da formação do tecido de granulação pelo ácido bio hialurônico e outros componentes de sua fórmula.

Com a pomada HYCOS o paciente pôde ser desopitalizado mais precocemente e dar continuidade ao seu tratamento da ferida de forma ambulatorial.

\section{REFERÊNCIAS}

1. Founier AJ. Etude clinique de la gangere foudroyante de la verge. Semaine Med 1884; 4:69.

2. Dornelas MT, Correa MPD, Barra FML, Corrêa LD, Silva EC, Dornelas GV. Fournier's syndrome: a 10-year evaluation study. Rev. Bras. Cir. Plást. Dec 2012; 27(4). DOI: https://doi.org/10.1590/S1983-

51752012000400022. Acesso em: 12 mar. 2021.

3. Custodio SAAS, Bruno FN, Spaziani AO, Pereira MZO, Diogo LF, Frota RS, Larrossa FM, Pinheiro DC, Faidiga L. Síndrome de fournier: análise de um relato de caso. Braz. J. Hea. Rev. jul./aug. 2020;3(4):7569-77. DOI: 10.34119/bjhrv3n4030. Acesso em: 12 mar. 2021.

4. Kılıç A, Aksoy Y, Kılıç A. Fournier's gangrene: etiology, treatment, and complications. Ann Plast Surg, 2001; 47(5):523-7.

5. Fahal AH, Hassan MA. Fournier's gangrene in Khartoum. $\mathrm{Br} \mathrm{J}$ Urol. 1988 May;61(5):451-4. DOI: $10.1111 /$ j.1464- 
410x.1988.tb06596.x. Acesso em: 12 mar. 2021.

6. Riseman JA, Zamboni WA, Curtis A. Graham DR, Konrad HR, Ross DS. Hyperbaric oxygen therapy for necrotizing fasciitis reduces mortality and the need for debridements. Surgery 1990 Nov;108(5):84750 .

7. Moses AE. Necrotizing fasciitis: flesheating microbes. Israel J Medical Sciences; 1996.

8. Laboratório Biotecnológico - TCI [Internet] HYCOS: Linha de produtos voltados ao combate de afecções dermatológicas, compostos de ativos naturais e componentes biotecnológicos com grau zero de toxicidade.
Disponível em: https://tcilab.com.br/. Acesso em: 05 abr 2021.

9. Chen SY, Fu JP, Chen TM, Chen SG. Reconstruction of Scrotal and Perineal Defects in Fournier's gangrene. J Plast Reconstr Aesthet Surg. 2011 Apr;64(4):52834. DOI: 10.1016/j.bjps.2010.07.018. Epub 2010 Aug 24.

10. Ellabban MG, Townsend PL. Singlestage Muscle Flap Reconstruction of Major Scrotal Defects: Report pf Two Cases. Br J Plast Surg. 2003 Jul;56(5):489-93. DOI: 10.1016/s0007-1226(03)00178-4.

Submissão: 2021-11-04

Aprovado: 2021-11-15 